\title{
Incidence \\ de la salinité sur les cultures irriguées
}

\author{
par J. Delmas, A. Grauby \\ C.E.A.-DPr/SRTE, Laboratoire de Radioécologie continentale, \\ C.E.N./Cadarache \\ ef C. Gleizes \\ Agence de Bassin Rhône-Méditerranée-Corse
}

L'industrialisation des vallées du Rhône et de ses principaux affluents, les possibilités d'exploitation d'importantes couches sédimentaires de chlorure de sodium, laissent prévoir un accroissement des rejets de sels dans les eaux superficielles. Ce risque avait été particulièrement souligné par la Commission de l'Eau lors de la préparation du $V^{e}$ Plan.

Parmi les usages de l'eau susceptibles d'être gênés par la salinité, figurent les irrigations, tout spécialement à l'aval de la vallée du Rhône, où plus de $100 \mathrm{~m}^{3} / \mathrm{s}$ ont été réservés à cette fin.

C'est la raison pour laquelle l'Agence de Bassin RhôneMéditerranée-Corse avait inscrit à son premier programme d'intervention l'étude des incidences de la pollution saline des eaux du Rhône sur le rendement des cultures irriguées. Cette étude, conduite en liaison avec le ministère de l'Agriculture, a été confiée au Département de Protection dı C.E.A. et à la Compagnie d'Aménagement de la Région du Bas-Rhône et du Languedoc.

C'est ainsi que le laboratoire de Radioécologie du Département de Protection implanté au C.E.N. de Cadarache a mis au point et exploité divers dispositifs expérimentaux destinés à mettre en évidence et à mesurer les différences de comportement de végétaux cultivés alimentés en eau plus ou moins chargée en sels.

\section{Programme d'étude}

Les incidences que peut avoir la pollution saline des eaux du Rhône sur le rendement des cultures résultent de phénomènes de divers types. L'augmentation de la pression osmotique de la solution du sol entraîne un accroissement de l'énergie nécessaire à la plante pour absorber l'eau du sol. Certains ions ont un effet toxique. Au cours de l'aspersion, l'évaporation sur le feuillage peut provoquer des brûlures. Certains ions, comme le sodium, ont un effet néfaste sur la structure des sols.

Ce sont les deux premiers effets qui ont fait l'objet d'une expérimentation par le Laboratoire de Radioécologie.

Cette expérimentation a pour but d'évaluer la baisse de rendement d'une culture dans les conditions d'irrigation actuelles, c'est-à-dire en l'absence du lessivage et du drainage utilisés habituellement pour lutter contre les excès de sels, et donc en tenant compte des accumulations de sel pendant la saison d'arrosage.

Les travaux déjà effectués en divers pays portent, en général, sur des teneurs supérieures à $2000 \mathrm{mg} / 1$. Les teneurs de 200 à $500 \mathrm{mg} / \mathrm{l}$, rencontrées dans l'eau du Rhône, correspondent alors à l'eau de référence. Ces travaux ne permettent pas d'extrapolation vers les faibles salinités. Ils sont d'ailleurs, exceptés ceux faits aux PaysBas sur des teneurs faibles, orientés vers une recherche de l'utilisation optimale d'eaux salées.

Aussi, en raison des conditions actuelles de rejets salins et des perspectives prévisibles, on a admis une gamme d'étude de 0 à $1000 \mathrm{mg} / \mathrm{l}$ de chlorure de sodium dans l'eau d'irrigation. Un deuxième sel était prévu pour différencier effet toxique et pression osmotique: le sulfate de sodium.

De nombreuses espèces cultivées dans la région du Bas-Rhône et du Languedoc étant sensibles à la salinité, les tests ont été effectués principalement avec le haricot (contender) qui est une de ces espècos. Une laitue a été également utilisée.

La plante, soumise à l'irrigation, utilise la solution du sol résultant de l'équilibre sol-eau d'irrigation. Une solution de référence représentant la solution de sol-type a été déterminée par la Compagnie Nationale d'Aménage- 
ment du Bas-Rhône-Languedoc à partir de cinq sols caractéristiques de sa zone d'intervention.

Cette solution est une adaptation de solution de Arnon et Hoagland 1938 majeurs $\times 0,7$. Elle correspond aux sols peu calciques (concentration en $\mathrm{Ca}: 5,6$ milliéquivalent).

L'expérimentation a eu lieu en deux temps:

1. Une expérimentation sur solution nutritive a permis la recherche des relations existant entre la salinité de la solution et les principaux paramètres caractérisant le végétal et son milieu ambiant;

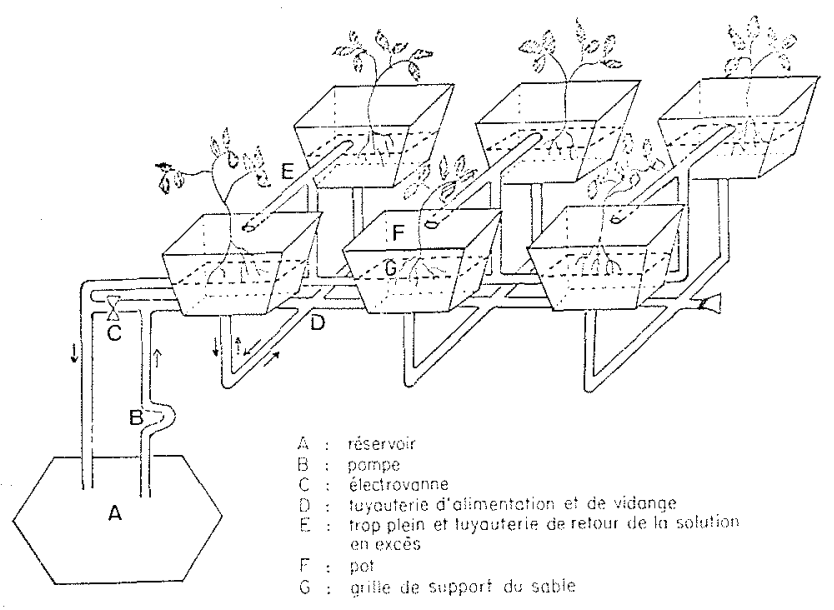

$1 /$ Dispositif de culture sur sable avec alimentation en solution nutritive par subirrigation pour étude de l'effet de la salinité sur les haricots.
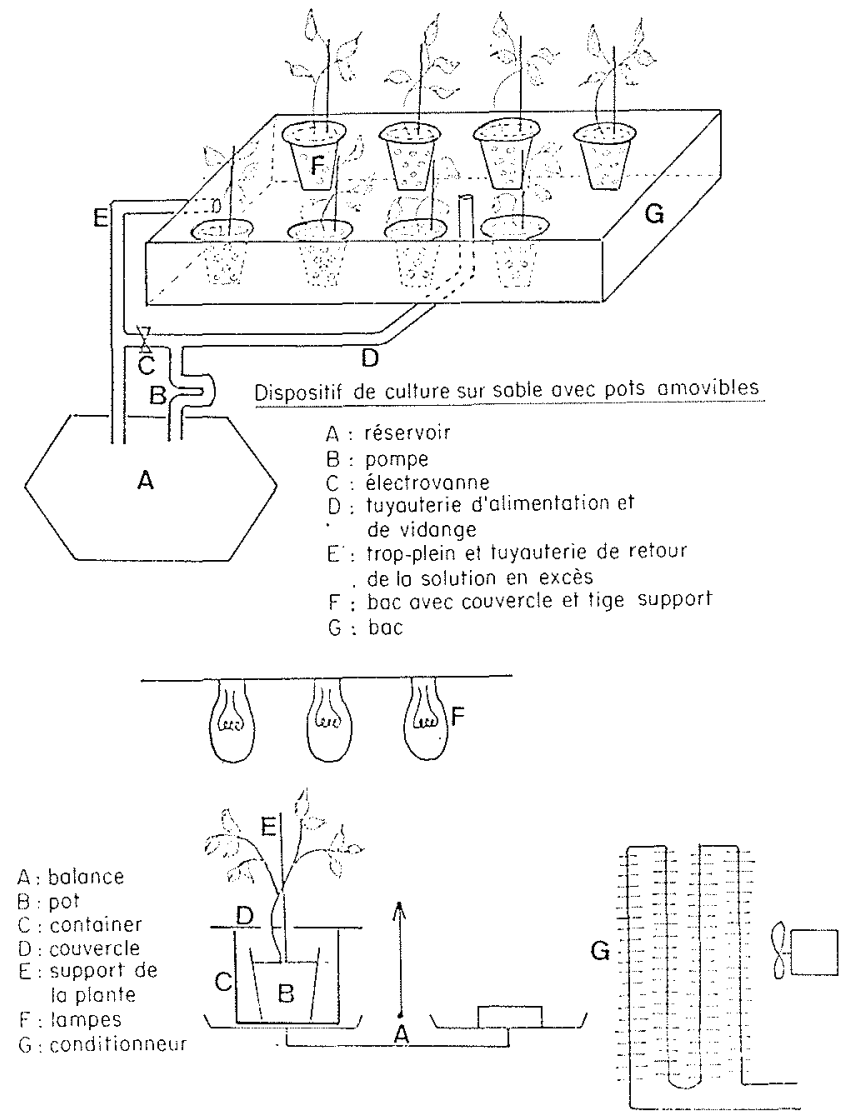

Dispositif de mesure de la consommation en eou por pesée

2/ Dispositifs pour l'étude

de l'effét de la salinité sur la consommation en eau des haricots.

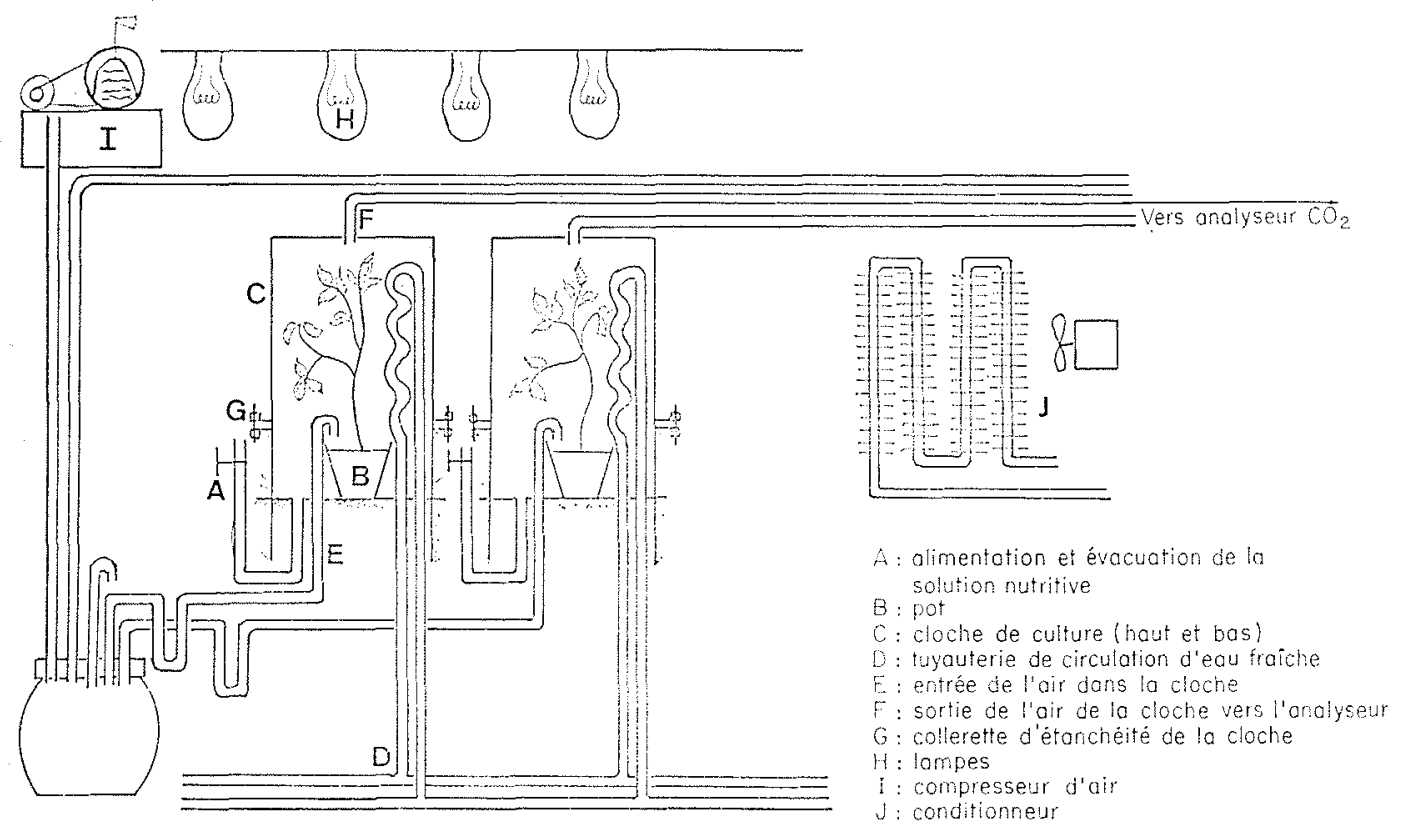

3/ Dispositif pour l'étude de l'effet de la salinité sur la pholosynthèse. 
2. Une expérimentation en petits lysimètres qui établit la liaison entre les essais sur solution et les essais sur sol, et a permis également de corréler rendement des cultures et salinité des eaux.

La difficulté de cette étude réside essentiellement dans la faible valeur des teneurs en sel à tester lorsque sont envisagées des eaux d'irrigation à bas niveaux de salinité et les réactions de la plante dans la première partie de son développement.

La méthode de culture sur sable avec pesée des productions ne permettant pas d'explorer les très bas niveaux, d'autres méthodes ont été envisagées. Les mesures d'absorption d'eau et de gaz carbonique sur des individus isolés et pendant un temps court ont permis de le faire en éliminant les différences existant entre individus.

\section{Les techniques}

Les essais sur solution nutritive et sur sols ont été effectués dans $70 \mathrm{~m}^{2}$ de serre dont les installations de conditionnement d'air stabilisaient la température à $25^{\circ} \mathrm{C}$ et l'hygrométrie à $50 \%$ sans éclairage d'appoint. Pendant l'été, par fort ensoleillement dans le milieu de la journée, la température s'est élevée à $30^{\circ} \mathrm{C}$ et l'hygrométrie a atteint 20 à $30 \%$. L'intensité lumineuse varie avec les saisons et en cours de journée. A titre d'exemple, le 19 juillet 1973, l'énergie totale reçue en 12 heures $17 \mathrm{mi}$ nutes a été de $813,6 \mathrm{~J} / \mathrm{cm}^{2}$.

\section{Culture sur sable. Dispositif avec pots.}

Le dispositif est présenté sur la figure 1. Sa particularité essentielle réside dans le sens de circulation de la solution nutritive dans les pots de bas en haut. Seize dispositifs semblables ont été utilisés au cours des différents essais.

\section{Culture en pots amovibles ef méthode de mesures de la consommation d'eau}

Les plants de haricots ont été élevés en godets indépendants, garnis de sable, disposés dans un bac lui-même rempli de sable (fig. 2). Le principe de l'alimentation en solution nutritive est voisin du dispositif précédent.

Une fois par semaine, pendant cinq semaines, chaque godet a été retiré de son bac, placé dans un bécher, rempli de la solution objet de l'essai et installé dans un phytotron pendant 12 heures. Un couvercle par godet limitait l'évaporation de la solution.

Les conditions maintenues dans le phytotron étaient les suivantes :

$$
\begin{aligned}
& \text { Température } \ldots \ldots \ldots \ldots \ldots \ldots \\
& \text { Hygrométrie } \ldots \ldots \ldots \ldots \ldots \ldots .50 \% \\
& \text { Intensité lumineuse } \ldots \ldots \ldots \ldots .40 \mathrm{~J} / \mathrm{cm}^{2} / \mathrm{h}
\end{aligned}
$$

L'absorption d'eau par les plantes a été mesurée par pesée.

Les effets d'alternance par changement des concentrations en $\mathrm{Cl}^{-}$des solutions ont été observés également.

\section{Culture sous cloches Mesure de l'absorption du $\mathrm{CO}_{2}$}

Chaque plante a été élevée sur sable en pot avec renouvellement des solutions nutritives deux fois par jour.

Les plantes sont placées sous cloches balayées par de l'air dont la teneur en $\mathrm{CO}_{2}$ est mesurée à l'entrée et à la sortie au moyen d'un chromatographe Lambert 16 voies (fig. 3).

\begin{tabular}{|c|c|}
\hline Température ............. & $28^{\circ} \mathrm{C}$ \\
\hline Hygrométrie & $75 \%$ \\
\hline Intensité lumineuse & $50 \mathrm{~J} / \mathrm{cm}^{2} / \mathrm{h}$ \\
\hline Durée d'éclairage journalier & 14 heures \\
\hline
\end{tabular}

Ces cloches, au nombre de 12 , sont disposées dans une chambre climatique. Dans les cloches les conditions étaient:

\section{Culture sur sol en lysimètres}

Sur sol, les effets de la salinité ont été testés dans quatre petits lysimètres de $1 \mathrm{~m}^{2}$ de surface et $70 \mathrm{~cm}$ de haut (hauteur de sol utile $50 \mathrm{~cm}$ ) (fig. 4).

Le sol a été mis en place horizon par horizon avec soin de façon à conserver au mieux la structure d'origine.

L'humidité du sol a été mesurée au moyen d'une sonde à neutrons. Le profil de l'humidité représentée par les taux de comptage a été déterminé tous les trois jours. Le profil de l'humidité critique a été évalué au préalable par approximation. Les arrosages ont été déclenchés lorsque ce profil d'humidité critique était atteint.

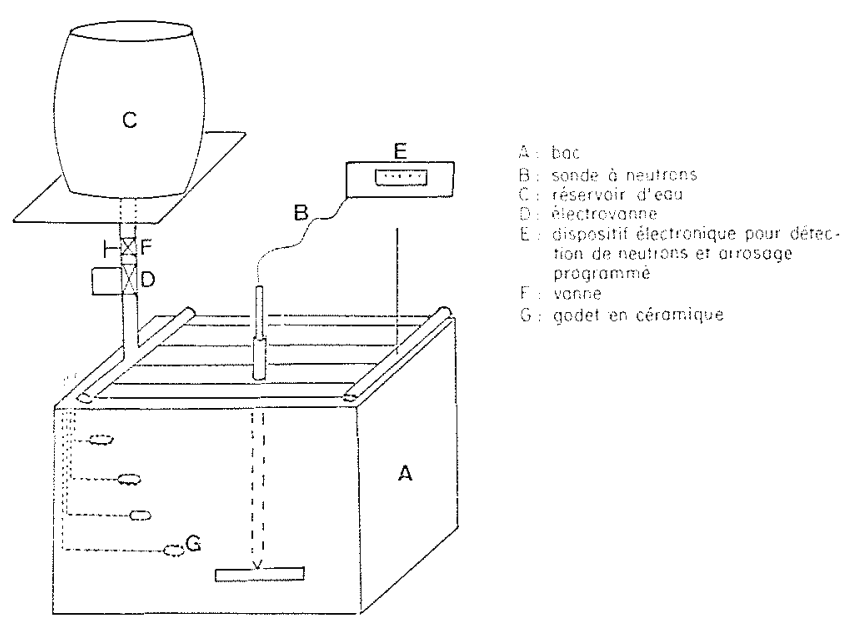

4/ Lysimètre pour l'étude des effets de la salinité de l'eau d'arrosage sur le haricot. 


\section{Effets de la salinité de la phase liquide du sol sur la plante}

Pour cette étude, le haricot, variété contender, a été choisi en raison de sa grande sensibilité au CINa. En effet, dans les tables de tolérances établies par le laboratoire d'étude de la salinité de Riverside, Californie (U.S.A.), le haricot est classé dans les espèces ne pouvant tolérer qu'une faible salinité [1]. Nous avons contrôlé cette sensibilité par rapport à la salade qui est moyennement tolérante. Dans cet essai la chute de production du haricot a été 1,4 fois plus forte que celle de la salade à $0,75 \mathrm{~g} / 1$. D'autres manifestations physiologiques se traduisant par une perturbation dans les équilibres ioniques ont confirmé cette réaction. Le haricot présente donc un grand intérêt pour mettre en évidence les réactions des plantes à de faibles variations de salinité.

- Toutes les parties de la plante sont affectées par le CINa, mais leur sensibilité est variable. Les feuilles de haricot sont plus sensibles que les gousses (fig. 5).

Pour des raisons pratiques, quelle que soit la culture considérée, il est plus intéressant d'envisager les problèmes de salinité vis-à-vis de la partie de végétal commercialisée. De ce fait, le poids des gousses récoltées a été choisi dans ces travaux comme indicateur de la réaction des haricots.

- Il est essentiel de connaître l'ensemble des caractères de l'environnement (naturel et agricole) afin de déterminer la nature du premier facteur limitant.

L'hiver, l'éclairement, constituant le facteur limitant principal l'effet de la salinité sur la production de gousses, est faible. En mars et juin l'éclairement intensif augmente la sensibilité des plantes à la salinité qui constitue alors le facteur limitant prépondérant (fig. 6).

On ne peut que recommander une grande prudence pour l'évaluation des baisses de rendement dues à la salinité, étant donné les influences prépondérantes des facteurs d'environnement et d'équilibre. Dans toute application pratique, la connaissance de ces facteurs semble indispensable, tout au moins pour une évaluation précise.

- Les travaux effectués à une pression osmotique supérieure à deux atmosphères ont montré que la nature des sels entrainant l'accroissement de la pression osmotique n'influait en rien sur le rendement végéta! [2]. Nos expériences ont donné des résultats différents à des niveaux de pression osmotique inférieurs à 2 . En effet, si le facteur d'accroissement est la concentration de sels nutritifs, le rendement croît avec la pression osmotique, alors que, si le facteur générateur est le polluant le rendement décroît (plus fortement pour le $\mathrm{ClNa}$ que pour le $\mathrm{SO}_{4} \mathrm{Na}_{2}$ ).

- Les plantes n'ont pas la même sensibilité aux différentes périodes de leur développement.

La plante est plus sensible au ClNa dans le premier tiers de son développement, c'est-à-dire entre l'apparition des premières feuilles non cotylédonaires et le début de la floraison. Les effets sur la production doivent être estimés en fonction de la concentration en
$\mathrm{Cl}^{-}$de la solution du sol pendant cette phase de la vie de la plante.

Du point de vue de la pratique agricole, cette observation conduit à surveiller de très près la salinité des eaux distribuées aux plantes dans la première partie de leur développement.

\section{Relation entre \\ la salinité (CINa) de l'eau d'irrigation et la production végétale}

Dans la pratique, l'eau d'arrosage est distribuée en cours de culture de façon à maintenir dans le sol une humidité favorable à la croissance des plantes. L'humidité du sol est maintenue ainsi entre la capacité au champ et l'humidité oritique. Les quantités d'eau d'appoint distribuées à cet effet sont conditionnées par le type de sol, la culture considérée, la rotation, la répartition des arrosages en cours de culture et la pluviométrie.

L'eau s'évapore, ou est absorbée par la plante, en cours de culture, et la concentration en $\mathrm{Cl}^{-}$du sol croît proportionnellement aux apports de sel qui sont déterminés par la concentration en $\mathrm{Cl}$ de l'eau ainsi que par les volumes distribués.

Les infiltrations éventuelles en profondeur, hors de portée des racines, sous l'effet des eaux excédentaires, limitent l'augmentation de la salinité du sol. Ce procédé est utilisé pour éliminer les excès de teneur en $\mathrm{Cl}$ du sol.

Dans nos essais, $1901 / \mathrm{m}^{2}$ ont été distribués pendant la croissance du haricot. Les irrigations ont été déclenchées à un taux d'humidité de $70 \%$ de la capacité au champ.

Un premier arrosage, avec 20 litres d'eau salée, a été apporté à un taux d'humidité du sol de $85 \%$ au stade d’apparition des premières feuilles non cotylédonaires.

Des eaux d'irrigation aux concentrations en $\mathrm{Cl}^{-}$suivantes ont été utilisées:

$$
0,030 \mathrm{~g} / 1-0,28 \mathrm{~g} / 1-0,530 \mathrm{~g} / 1-1,03 \mathrm{~g} / 1-2,03 \mathrm{~g} / 1 \text {. }
$$

Sous l'effet de ces arrosages, les concentrations en ions $\mathrm{Cl}^{-}$des solutions du sol des couches exploitées par les racines croissent en rapport des quantités d'eau et de sel apportées.

Ces concentrations en $\mathrm{Cl}$, aux différents stades de développement des plantes, ont été déterminées par prélèvement de la solution du sol au moyen de godets en céramique.

Sur le graphique 7, les effets sur le haricot, en $\%$ du témoin, de la salinité (en $\mathrm{g} / \mathrm{l}$ de $\mathrm{Cl}$ ) de la phase liquide, où se développe les racines, sont représentés par deux droites de régression correspondant aux différents essais.

- Une droite représente la relation entre la concentration en ions $\mathrm{Cl}^{-}$de la solution du sol et la production de gousses.

Etant donné la sensibilité du haricot pendant la première partie de son développement, les productions de gousses obtenues au cours de trois séries d'essais sur sol en lysimètres sont reliées aux teneurs en $\mathrm{Cl}$ moyennes de la solution du sol pendant cette période. 
- Une autre droite représente les effets de la salinité sur la production obtenue sur solution nutritive.

Les relations de la figure 7 , entre la production de gousses et les concentrations en $\mathrm{g} / 1$ de $\mathrm{Cl}$ des solutions nutritives et de la phase liquide des sols des lysimètres, sont voisines. Les pentes sont pratiquement les mêmes.

Les observations en culture sur solution nutritive sur l'effet du CINa sur le haricot peuvent donc être considérées comme représentatives de la réaction à la salinité de cette plante cultivée sur sol. Les incidences des caractéristiques de l'environnement sur la production de cette plante, observées dans ces conditions, peuvent être également considérées comme vraisemblables. Ces observations présentent d'ailleurs une grande analogie avec celles que Bierhuizen a pu faire aux Pays-Bas sur les cultures irriguées de serre.

On peut également considérer comme plausibles les différences de comportement des diverses parties du végétal et de l'absorption d'eau. Nous avons vu, en effet, que la plante absorbe moins d'eau dès les plus bas niveaux de concentration en CINa. Cependant, pour des raisons pratiques, il est intéressant de considérer de préférence les réactions de la partie du végétal qui a une importance économique.

En définitive, nous adopterons la relation obtenue sur solution nutritive (1). Elle est, en effet, plus représentative que celle qui a été obtenue sur sol. Elle a été déterminée à partir des extraits de pâtes saturés de 5 sols représentatifs de la région du Bas-Rhône et du Languedoc. Les concentrations en $\mathrm{Cl}$ des solutions sont connues de façon précise.

$$
Y=-76,8 X+105,7 \quad(r=-0,73)
$$

Rappelons que :

$Y$ est la production en $\%$ du témoin à salinité 0 ;

$X$ est la concentration en $\mathrm{Cl}$ en $\mathrm{g} / \mathrm{l}$ de la phase liquide (solution du sol à la capacité au champ et solution nutritive) moyenne des concentrations comprises entre l'apparition des premières feuilles non cotylédonaires et du début de la floraison.

Cette loi donne une représentation moyenne de l'effet de la salinité de la solution du sol sur la production de gousses du haricot. Elle n'est valable que dans certaines limites en raison de l'action des divers paramètres de croissance.

Elle a été établie en prenant en compte les concentrations en $\mathrm{Cl}$ allant de $0,25 \mathrm{~g} / 1$ à $0,75 \mathrm{~g} / 1$.

Nous avons irrigué en deux fois pendant la première partie du développement de la plante, période de sensibilité maximum de celle-ci. Une première fois: 20 litres d'une eau dont la concentration était de $C_{c}$ à l'apparition de la première feuille non cotylédonaire.

Une deuxième fois: 40 litres de la même eau à mitemps entre l'apparition de la première feuille non coty. lédonaire et le début de la floraison. Si l'on désigne par $C_{s}$ la concentration en $\mathrm{Cl}$ en $\mathrm{g} / \mathrm{l}$ de la solution du sol à la capacité au champ avant arrosage et par $\Delta C_{s}$ l'apport global du $\mathrm{Cl}$ en $\mathrm{g} / \mathrm{l}$ dans cette même solution du sol par les arrosages pendant la période de sensibilité, il est clair que la concentration moyenne dans la période de sensibilité maximum de la plante est de:

$$
X=1 / 2\left[C_{s}+1 / 3 \Delta C_{s}+C_{s}+3 / 3 \Delta C_{s}\right]
$$

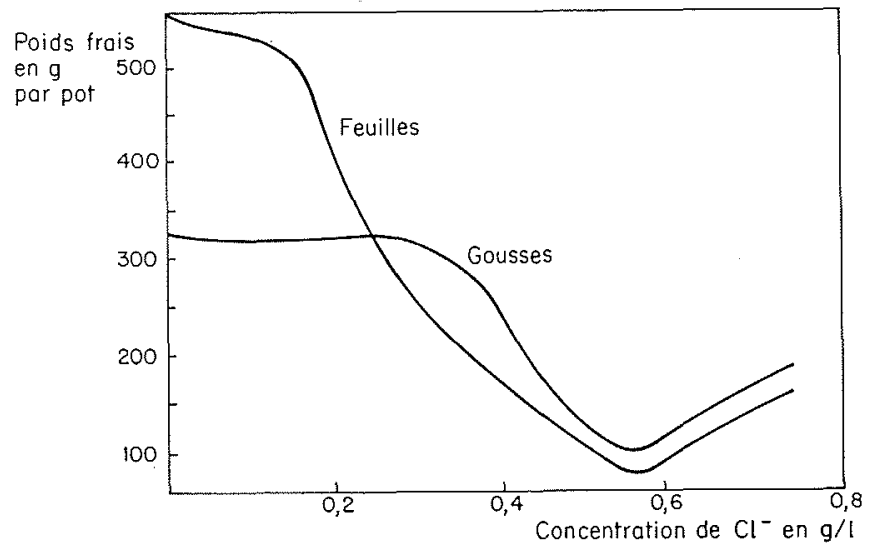

5/ Sensibilité des feuilles et des gousses de haricot à la salinité des solutions nutritives (en $\mathrm{g} / \mathrm{l}$ de $\mathrm{Cl}$ ).

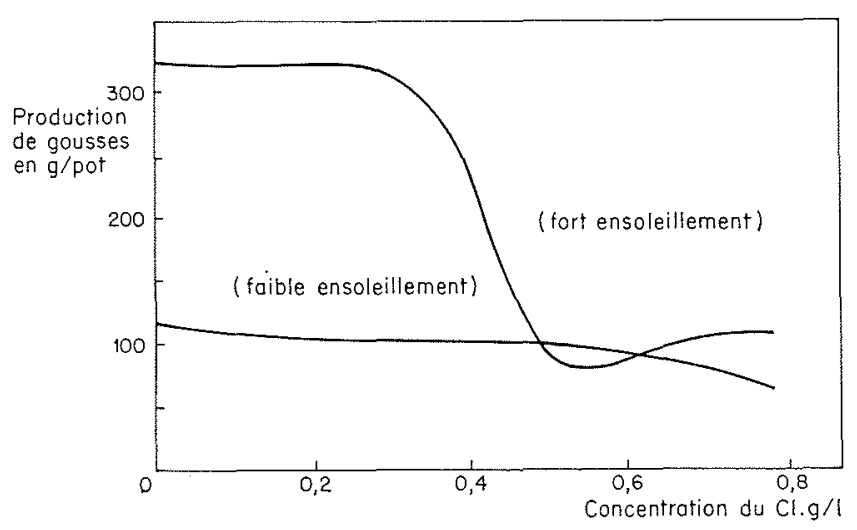

6/ Effet de la salinité des solutions nutritives (en $\mathrm{g} / / \mathrm{de} \mathrm{Cl}$ ) sur la production de gousses en novembre-décembre et mal-juin.

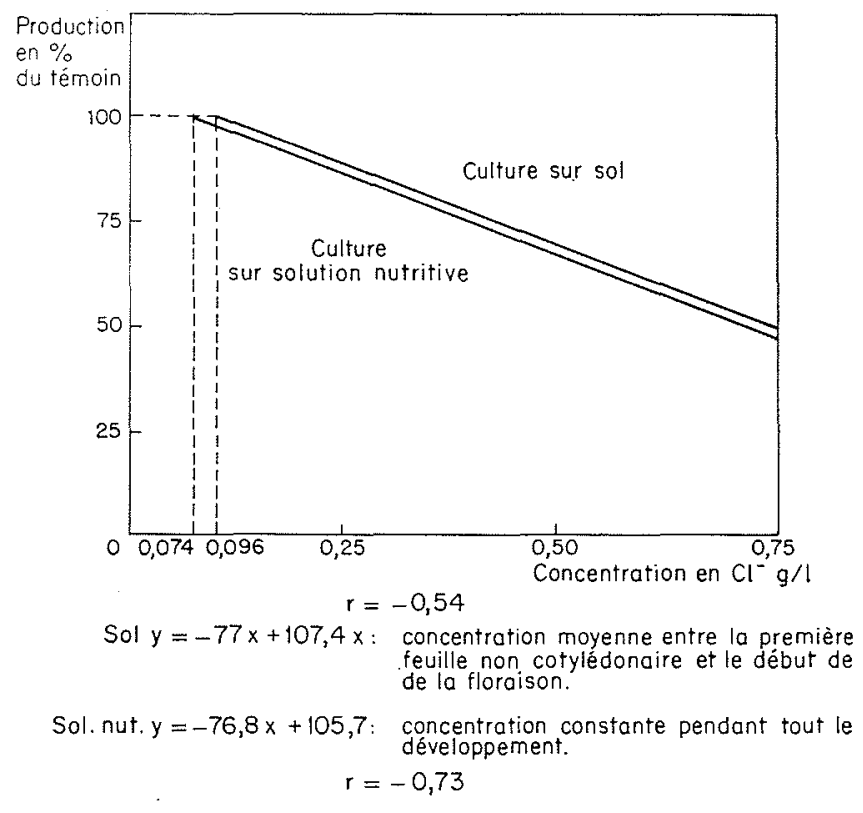

7/ Effet de la salinité (en $\mathrm{g} / \mathrm{l}$ de $\mathrm{Cl}$ ) des solutions nutritives ef de la solution du sol sur la production de gousses du haricot. 
L'apport de $\mathrm{Cl}$ en $\mathrm{g} / \mathrm{I} \Delta C_{s}$ dû à l'arrosage est fonction de la concentration de l'eau $C_{e}$ du volume d'eau $V$, de l'humidité à la capacité au champ $H_{c}$, de la profondeur de sol exploité par les racines $P$ :

$$
\Delta C_{s}=V \cdot C_{o} / P \cdot H_{c}
$$
tal :

A titre d'exemple, soit les valeurs du sol expérimen-

$$
\begin{gathered}
H_{c}=29 \begin{array}{cc}
P=5 \mathrm{dm} & C_{s}=0,092 \mathrm{~g} / \mathrm{en} \mathrm{Cl} \\
C_{e}=0,2 \mathrm{~g} / 1 & X=0,147
\end{array} \\
Y=94,4 \%, \text { soit une baisse de rendement de } 5,6 \%
\end{gathered}
$$

En extrapolant pour $\mathrm{Y}=100$, la valeur de $\mathrm{X}$ est $0,074 \mathrm{~g} / \mathrm{l}$; ce qui donne à penser à l'existence d'un seuil où le haricot ne serait pas affecté par la salinité.

En fait, aucun des résultats trouvés au cours des essais mettant en œuvre des méthodes de mesures sensibles de la réaction du haricot (absorption d'eau et gaz carbonique) ne permet d'affirmer l'existence de ce seuil.

Des auteurs hollandais [3] [4] ont observé des lois semblables mais avec des pentes moins fortes. Dans leurs essais sur le concombre, une augmentation de $100 \mathrm{mg} / 1$ de la concentration en $\mathrm{Cl}$ moyenne de la solution du sol, pendant toute la durée de la culture en serre dans leur région, ne fait baisser la production que de 3,5\%, deux fois moins que pour les gousses de haricot en région méditerranéenne.

Cette réponse plus accentuée de la production à la salinité est difficilement appréciable, compte tenu des conditions des études qui sont essentiellement différentes (région, espèce végétale, etc.). Nous ne retiendrons simplement que l'aspect linéaire.

Pour les valeurs supérieures à $0,75 \mathrm{~g} / 1$, on a de bonnes raisons d'adopter encore une relation linéaire jusqu'à environ $2 \mathrm{~g} / 1$, mais de décroissance plus faible : -21 contre $-77$.

Ces représentations linéaires donnent une enveloppe globale du phénomène puisque, en étudiant précédemment les effets de chaque paramètre retenu, nous avons mis en évidence des réactions inexpliquées.

\section{Conclusion}

- Il est possible de prévoir l'effet de la qualité de l'eau d'irrigation sur la production d'un végétal.

- Dans l'étude que nous venons de faire, nous avons choisi le haricot comme espèce végétale en raison de sa grande sensibilité au $\mathrm{CINa}$.

- Les effets du polluant sur la production du haricot ont été les mêmes en solution du sol ou en solution nutritive. Ce résultat a permis de travailler sur solution nutritive qui présente des avantages de commodité et de précision, et qui, de plus, est représentative des solutions des sols locaux.

- L'étude séparée des différents paramètres pouvant intervenir sur l'effet de la salinité sur le haricot a donné des résultats très variés :

- Les diverses parties du végétal ont des sensibilités différentes au CINa. La feuille s'est révélée plus sensible que les gousses et les tiges;
- Un éclairement intensif augmente la sensibilité de la plante;

- Le stade de végétation où le haricot réagit le plus à l'apport de sel est la période qui s'écoule entre l'apparition de la première feuille non cotylédonaire et la floraison; on ne peut même pas assurer de la sensibilité du haricot après la floraison. Ceci peut intéresser particulièrement les agriculteurs pour orienter la surveillance des irrigations;

- Les travaux effectués à une pression osmotique supérieure à deux atmosphères ont montré que la nature des sels entraînant l'accroissement de la pression osmotique n'influait en rien sur le rendement végétal. Nos expériences ont donné des résultats différents à des niveaux de pression osmotique inférieurs à 2 . En effet, si le facteur d'accroissement est la concentration de sels nutritifs, le rendement croît avec la pression osmotique, alors que, si le facteur générateur est le polluant, le rendement décroît (plus fortement pour le $\mathrm{CINa}$ que pour le $\mathrm{SO}_{4} \mathrm{Na}_{2}$ ).

- On ne peut que recommander une grande prudence pour l'évaluation des baisses de rendement dues à la salinité, étant donné les influences prépondérantes des facteurs d'environnement et d'équilibre. Dans toute application pratique, la connaissance de ces facteurs semble indispensable, tout au moins pour une évaluation précise.

- Une évaluation globale de la baisse de rendement due au polluant pout être représentée par un modèle linéaire. $\mathrm{Ce}$ modèle n'est probablement plus très fidèle pour les faibles concentrations.

- Pour l'étude de l'effet de la salinité dans ces zones on a mis en œuvre deux méthodes d'expérimentation permettant l'observation des effets du CINa à partir de l'absorption de l'eau et de la photosynthèse. Nous n'avons pu mettre en évidence de seuil de salinité.

- Pour les fortes concentrations de $\mathrm{Cl}$, entre $0,75 \mathrm{~g} / 1$ et $2 \mathrm{~g} / 1$, il semblerait que les effets dépressifs soient moindres.

- La salinité de l'eau d'irrigation et la salinité de l'eau du sol sont également liées par un modèle linéaire.

Il y a done linéarité entre la production de gousses du haricot et les concentrations en $\mathrm{Cl}$ de l'eau d'irrigation, ce qui permet effectivement de prévoir l'effet du polluant sur la production du végétal.

\section{Bibliographie}

[1] U.S. Salinity Laboratory. - Diagnosis and improvement of saline and alkali soils, U.S.D.A. Agriculture Handbook $\mathrm{n}^{\circ} 60$ (1954).

[2] Gouny (P.) et Brachet (J.). - Bulletin Technique d'Information, $\mathrm{n}^{\circ} 224$ (novembre 1967).

[3] BrerhuizeN (J.F.). - Water quality and yield depression. Technical Bulletin, $\mathrm{n}^{\circ}$ 61, Wageningen, The Nether!ands (1969).

[4] Ploegman (C.) en Bierhuizen (J. F.). - « Zonttolerantie van Komkommer», Instituat voor Cultubrtechnick en Waterhwishouding m-dedeling 126 (1970).

[5] UNESCO-UNDP-CSF. - Tech. Rpt: « Recherche en matière d'irrigation avec des caux salées ». TUN. 5, Paris (août 1970). 


\title{
Discussion
}

\author{
Président : M. C. LEFrou
}

M. le Président remercie M. Delmas de son exposé fort bien illustré et souligne le grand intérêt de ce type d'étude qui a pour but de déterminer la qualité de l'eaul nécessaire pour un usage déterminé; ce point est essentiel pour une bonne gestion des ressources en eall. Il ouvre ensuite la discussion.

M. BALdY (I.N.R.A.) présente les deux remarques suivantes :

$1^{\circ}$ Vous avez étudié les rendements sur les poids frais des végétaux; d'après mon expérience des problèmes de salinité en Afrique du Nord les teneurs en matières sèches des haricots ont dû varier avec la salinité.

$2^{\circ}$ Les valeurs de l'éclairement sur vos cultures m'ont paru faibles par rapport à celles observées en p'ein champ. Il est vrai que le haricot a des exigences énergétiques réduites par rapport à d'autres plantes.

La réponse de $M$. Delmas sur ces deux points peut être résumée comme suit :

$1^{\circ}$ Nous nous sommes attachés à l'étude des rendements en poids du végétal frais car il nous a paru le plus représentatif au point de vue commercial; mais l'étude des matières sèches est certainement d'un grand intérêt.

$2^{\circ}$ Les essais ont été effectués dans deux types différents d'instal'ations :

- d'une part, une serre à parois vitrées, exposée au rayonnement solaire, à l'intérieur de laquelle l'éclairement était de la moitié aux deux tiers de l'éclairement extérieur,

- d'autre part, de chambres climatiques avec éclairage artificiel donnant un éclairement de 10000 à 20000 lux. Cet éclairement est très souvent employé dans les études de physiologie végétale.
M. LEMOINE (S.O.M.I.V.A.C.) souhaiterait savoir si, à l'occasion des recherches présentées par le conférencier, il a été procédé à une étude de l'influence de la salinité sur la qualité du produit, des analyses de végétaux ont-elles été effectuées pour tenter de déter miner la variation de leur teneur en chlorures en fonction de la salinité de la solution d'alimentation?

\section{Delmas répond :}

ll serait trop long de détailler toutes les observations effectuées et nous avons dû nous borner à l'essentiel. Signalons pourtant qu'il y a une corrélation positive très bonne entre la concentration en chlore de la phase liquide et du tissu du haricot; plus la concentration en chlore crôt dans la solution, plus elle crô̂t dans le tissu du haricot. Nous avons pu constater, dans le cas de la salade qui est relativement plus résistante à la salinité - de bonnes corrélations négatives entre le chlore, le calcium, le potassium et le magnésium.

Effectivement, il y a accumulation de chlore dans les tissus végétaulx. Dans le cas d'une espèce comme l'oranger dont les feuilles subsistent sur l'arbre pendant deux à trois ans, il est possible que l'on puisse constater une accumulation de chlore très marquée. Nous travaillons sur l'oranger avec la station de San Giulian, qui a constaté des phénomènes analogues portant, non pas sur le chlore, mais sur de strontium. En vieillissant, les feuilles accumulent très fortement le strontium et cela amène des taux de transfert ultérieurs dans les fruits assez importants.

M. le Président clôt la discussion et donne la parole à M. DurousSEAU pour l'exposé de sa communication.

\section{Abstract}

\section{Effects of salinity on irrigated crops}

The effects of irrigation water quality on plant growth is predictable. The haricot bean was considered in this study, because of its high sensitivity to $\mathrm{NaCl}$, which was found to have the same effect on crop output in both soil and nutrient solutions. The study was limited to consideration of a nutrient solution, which is easier to handle, gives accurate results and is representative of local soil conditions.

Separate investigation of factors governing the effect of salinity on the haricot bean showed that :-

1. Leaves are more sensitive to $\mathrm{NaCl}$ than pods and stalks.

2. Plant sensitivity increases under bright illumination.

3. The stage of growth at which the p'ant reacts most strongly to salt is between the first seedless leaf and flowering. Afterwards, salt-sensitivity can no longer be determined. This will no doubt be of interest to farmers planning jrrigation.

4. At osmotic pressures in excess of 2 atmospheres, plant yield is totally unafiected by the sa't causing the osmotic pressure rise. Below 2 atmospheres, yield increases with osmotic pressure if the pressure rise is caused by nutrient salt concentration, but decreases if salts are responsible (especially $\mathrm{NaCl}$, and to a lesser degree $\mathrm{Na}_{2} \mathrm{SO}_{4}$ ).

Owing to the decisive effects of environmental and equilibrium factors, considerable caution is advisable when evaluating loss of yield due to salinity. These factors must be known for al practical evaluation purposes.

An overall evaluation of loss of yield due to salt can be simulated by a linear model, except at low concentrations, fo which this type of model is probably no longer representative.

The effect of salinity in the considered zones was studied by two experimental methods in which observation of the effects of $\mathrm{NaCl}$ was based on water absorption and photosynthesis. Attempts to determine a critical salinity value were unsuccessful.

High $\mathrm{Cl}$ concentrations $(0.75-2 \mathrm{~g} / 1)$ apparently produced less depressive effects.

Irrigation and soil water salinities were also re'ated by a linear model.

Hence, there is a linear relationship between haricot bean pod output and irrigation water $\mathrm{Cl}$ concentration, from which the effect of salt pollution on p'ant yield can be predicted. 